\title{
Condition Assessment of Power Cable for Incomplete Information Based on Probabilistic Neural Network
}

\author{
Erxia $\mathrm{Li}^{1}$, Yonghua $\mathrm{Fan}^{1}$, Chaoqun $\mathrm{Kang}^{1}$, Yuling $\mathrm{Li}^{1}$, Xinrui $\mathrm{Hao}^{2, *}$ \\ and Zheng Zhang ${ }^{2}$ \\ ${ }^{1}$ China Electric Power Science Research Institute, Beijing, China \\ ${ }^{2}$ School of Electrical Engineering, Wuhan University, China \\ ${ }^{2}$ haoxinrui2009@163.com
}

\begin{abstract}
A new assessment method based on the combination of factor analysis and probabilistic neural network (PNN) is proposed to meet the research of main equipment condition-based maintenance in power distribution network. As the quantity of parameters that represent the state of the cable is many, the multi-source information database is established. The missing parameters for incomplete information is eliminated, and the factor analysis is used to calculate the main factors of the database and their factor scores, and the probability neural network was used to evaluate the operation status of the cable, then obtain a condition assessment method of power cable based on the probability neural network. Simulation results show that the proposed method could assess the state of power cable condition for the incomplete information effectively and accurately, and also provide reliable guidance for the cable maintenance.
\end{abstract}

Keywords: condition assessment of power cable, incomplete information, multi-source information database, factor analysis method, probabilistic neural network

\section{Introduction}

Power cable is the key equipment in power system, whose health status directly affects the operation of transmission and distribution system safely and stably. Once a failure happened, it may cause an extended power outage, and may even lead to a chain reaction and failure of associated equipment, eventually other parts of power system become paralyzed. Therefore, it is very important to evaluate the health status of the power cable scientifically and effectively and to find the cable fault in time.

The state parameters characterizing the health information of power cable are numerous, including state information, operation information, and inspection information, which belong to multi-category information sources. Thus it is great significance to create multi-source information database of cables. At present, there are many kinds of information database according to the different classification methods of cable state parameters. Ref. [1] analyzed the defects of power cable and designed a state evaluation framework, in which the state parameters are classified according to the type of defects. While in Ref. [2] the state parameters was classified according to the on-line monitoring data. This paper probed into the development of power cable insulation online monitoring technology, but which did not take into account status information and operation information. Authors in Ref. [3] established a multi-level index system of cable state evaluation in accordance with the division of different cable parts, but they did not put forward feasible measures for the condition assessment when information was incomplete.

Received (September 7, 2016), Review Result (October 13, 2016), Accepted (November 21, 2016) 
In conclusion, the research of multi-source information database on cable status can be summarized as: (1) the state variables contained in database were not comprehensive or not standardized; (2) the existence of information overlap in database make part of the state variables redundancy; (3) for incomplete information system, there is no suitable information fusion technology to multi-source information, and lack of complete cable condition assessment system.

Much researches associated with condition assessment methods of power cable have been done, such as fuzzy mathematical method [4-6], data mining method [7], analytic hierarchy process (AHP) [3], decision tree technology [8], D-S evidence theory [9] and measuring methods in view of specific characteristics [10-13]. But these evaluation methods will be subject to a certain subjective influence, and the status of the cable under the condition of incomplete information cannot be a scientific and effective evaluation.

Therefore, in this paper a multi-source information database of cable is established which has a fusion of many kinds of information, such as state information, operation information and inspection information; and a new assessment method of cable based on the combination of factor analysis and probabilistic neural network (PNN) is proposed. The results show that the proposed method could assess the state of power cable condition under the incomplete information effectively and accurately.

\section{Multi-Source Information Database}

\subsection{The Classification of State Information of Cables}

Both the reliable state information and the scientific evaluation method are the basis for carrying out the state evaluation. The comprehensive and reliable state information is an important prerequisite for accurately judging the health status of the cable, such as the aging degree and remaining life, and realizing the condition-based maintenance.

In this paper, the state information of cables is classified into three categories: equipment basic information, operation information, inspection information, family defects, as is shown in Table 1. The multi-source information database of the cable is established based on the state parameters listed in Table 1, combining with the actual operation history data of the cable.

Table 1. Division of Cable State Information

\begin{tabular}{cc}
\hline The category of state information & State parameters \\
\hline Equipment basic information & Rated voltage \\
& Rated current \\
Length of line & Wire section \\
\hline Operation information & Operating voltage \\
& Operating current \\
Loads \\
Operated life \\
\hline Inspection information & Partial discharge value \\
& Dielectric loss \\
& Conductor temperature of electric cable \\
& Insulation resistance of the main insulation \\
& Appearance of cable \\
& Cable joint temperature
\end{tabular}


Direct grounding current

Sheath protection side current

The loop current

Environment temperature

Environment humidity

\begin{tabular}{cc}
\hline Family defects & $\begin{array}{c}\text { Similar cable fault condition } \\
\text { Manufacturer's product quality statistics }\end{array}$ \\
\hline
\end{tabular}

\subsection{Incomplete Information and State Division}

In the practical operation of distribution network, some state information may be lost because of cable monitoring equipment fault and information record loss, then the multisource information database is not complete, which makes the cable comprehensive state evaluation greatly reduce in the accuracy and validity, even can not be assessed properly. Therefore, the case of incomplete information should be considered in the study of cable condition assessment.

According to the relevant guides [14], the state of the cable is divided into: normal state, attention state, abnormal state and serious state.

(1) Normal state: It is indicated that all the state of the cable are in stable condition, and within the alert value and the attention value (standard limit). The cable can be operated safely and normally.

(2) Attention state: It indicates that there is a trend toward in the direction of the change of some state parameters of the cable, which are close to the standard limit, but not more than the standard limit. While the power cable still can continue to run, monitoring should be strengthened at the same time.

(3) Abnormal state: It is indicated that some important state parameters of the cable are close to or slightly exceed the standard limit value. The cable should be operated under monitor and arranged examination reparation in time.

(4) Serious state: It is indicated that some important state parameters of the cable is more than the standard limit serious, so it is necessary to arrange the outage maintenance as soon as possible.

\section{Determine the Factor Score of the State Parameters Based on the Factor Analysis Method}

\subsection{Basic Principle of Factor Analysis Method}

Factor analysis is a technique to reduce dimension and simplify data in statistics, and it explores the basic structure of the observed data by studying the relationship among the variables, and uses a few "abstract" variables to represent the basic data structure. These abstract variables are called "factors", which can reflect the main information of many variables [15].

In factor analysis, the main factor is the common influence factor which can not be observed directly, but exist objectively. Each original variable $X_{i}$ can be expressed as the sum of linear function of main factor and the special factor as Equation (1).

$$
X_{i}=a_{i 1} F_{1}+a_{i 2} F_{2}+\cdots+a_{i m} F_{m}+\varepsilon_{i},(i=1,2, \cdots, p)
$$

Where $F_{1}, F_{2}, \ldots, F_{\mathrm{m}}$ are the main factors; $\varepsilon_{i}$ is the special factor; $A=\left(a_{i j}\right): p \times m$ is the factor load matrix. This model can be expressed as:

Where

$$
X=A F+\varepsilon
$$


Usually assumed that

$$
X=\left[\begin{array}{c}
X_{1} \\
X_{2} \\
\vdots \\
X_{p}
\end{array}\right], A=\left[\begin{array}{cccc}
a_{11} & a_{12} & \cdots & a_{1 m} \\
a_{21} & a_{22} & \cdots & a_{2 m} \\
\vdots & \vdots & \ddots & \vdots \\
a_{p 1} & a_{p 2} & \cdots & a_{p m}
\end{array}\right], F=\left[\begin{array}{c}
F_{1} \\
F_{2} \\
\vdots \\
F_{m}
\end{array}\right], \varepsilon=\left[\begin{array}{c}
\varepsilon_{1} \\
\varepsilon_{2} \\
\vdots \\
\varepsilon_{p}
\end{array}\right]
$$

$$
\left\{\begin{array}{l}
m \leq p \\
E(F)=0 \\
E(\varepsilon)=0 \\
V(F)=I \\
V(\varepsilon)=D=\operatorname{diag}\left(\sigma_{1}^{2}, \sigma_{2}^{2}, \cdots, \sigma_{p}^{2}\right) \\
\operatorname{Cov}(F, \varepsilon)=0
\end{array}\right.
$$

The principal component analysis method is usually used to solve the factor load matrix. As is shown in Equation (4)

$$
A=\left(\sqrt{\lambda_{1}} T_{1}, \sqrt{\lambda_{2}} T_{2}, \cdots, \sqrt{\lambda_{m}} T_{m}\right)
$$

Where $\lambda_{i}$ and $T_{i}$ are the eigenvalues and eigenvectors of the covariance matrix of the original data respectively.

Factor score is the final embodiment of factor analysis. The specific value of each factor in each sample can be calculated when the factor load matrix is determined, which is called factor score. After getting the factor score, you can use the factor score instead of the original variable. The formula for calculating the factor score is Equation (5).

$$
F=A^{\prime} \Sigma^{-1} X
$$

Then this method makes the sample containing $p$ state parameters reduce the dimension into a sample containing only $m$ main factors.

\subsection{The Application of Factor Analysis in Incomplete Information}

At present, the application of factor analysis method in the condition assessment of power grid equipment is mainly focused on the extraction of key parameters. This application is only to rank the importance of state parameters by the factor analysis method, not to find the "common influence factor" implied in parameters, namely the main factor. In addition, this kind of application only extracts the key parameters to carry on the state evaluation, and ignores the influence of the non-critical parameters, which will lead to the reduction of the accuracy of the evaluation result. According to the principle of factor analysis, it is possible to find out the common influencing factors of the state parameter, which makes it possible for the condition assessment of the cable under the condition of incomplete information.

Incomplete information will influence the accuracy and effectiveness of the condition assessment of power cable. According to the multi-source information database, it can be found that there is a certain correlation between state variables. For example, Partial discharge has influence on the dielectric medium, slight partial discharge has little effect on insulation, and dielectric strength decreased slowly, while intense partial discharge would cause the insulation strength decreased rapidly. At the same time, the value of insulation resistance of the cable can also directly reflect the dielectric strength. As a result, the three states included the partial discharge, the main insulation resistance and the insulation resistance of the outer shield reflects information of the strength of the power cable insulation, as shown in Figure 1(a). When one or two of the state information is missing, the remaining amount is still able to reflect the insulation strength of power cable, which reflects the overlap of the information in the three states. 


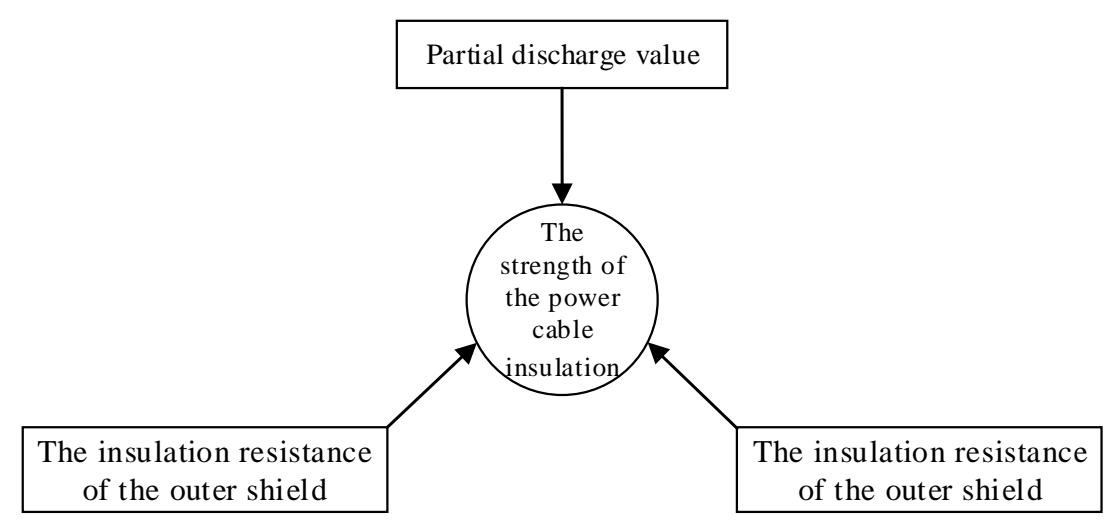

(a)

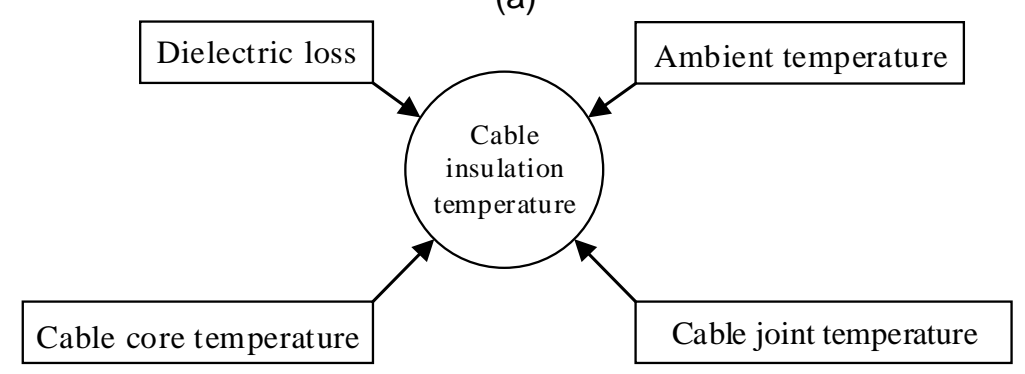

(b)

Figure 1. The Overlap of Information between States

Similarly, state variables included the dielectric loss, power cable core temperature, ambient temperature and the middle joint reflect the characteristic information of power cable insulation temperature, as shown in Figure 1(b). In addition, we can assume that the seven state variables included the partial discharge magnitude, main insulation resistance and the outer sheath of the insulation resistance, dielectric loss, temperature of cable, the middle joint and the temperature of the environment all reflect the state of the cable insulation material in different aspects.

Above discussion indicates that there is overlap between state variables information, when part of state variables information are missing, the remaining state variables may contain information of missing state variables. Therefore, the remaining state variables of power cable state information can reflect the missing state variables information, so as to realize the power cable condition assessment under incomplete Information.

According to the principle the main factor method on Section 2.1, factor analysis can overcome relevance, overlap, and extract fewer main factor to replace the original with more variables, which can both reflect the original with more variables information and reflect implied information of missing variables. For example, a main factor represents the cable insulation strength, which replaces the three original variables of the partial discharge, and the main insulation and outer sheath insulation resistance of insulation resistance, even if state variables information is missing. The extracted main factor still reflects the implied three cable insulation strength information. Thus, the factor analysis can be used for the power cable condition assessment for incomplete information.

When the state variables information is missing, the corresponding state variables in the multi-source information database is removed, and the main factor of the remaining state variables is extracted by factor analysis method. Due to the overlap of information between the state variables, the main factors can express the mis sing information. 


\section{A Comprehensive Assessment Method Based on Probabilistic Neural Network}

\subsection{Basic Principle of Probabilistic Neural Network}

In this paper, probabilistic neural network (PNN) method is proposed to assess the condition of power cable. PNN can be regarded as a radial basis neural network, which fuses density function estimation with Bayesian decision theory based on RBF network, and constructs the computing nonlinear discriminant boundary of probabilistic neural network, and the decision boundary is close to the optimal Bayesian decision surface [16].

The PNN is composed of the input layer, the hidden layer, the summation layer and the output layer. The structure of probabilistic neural network is shown in Figure 2.

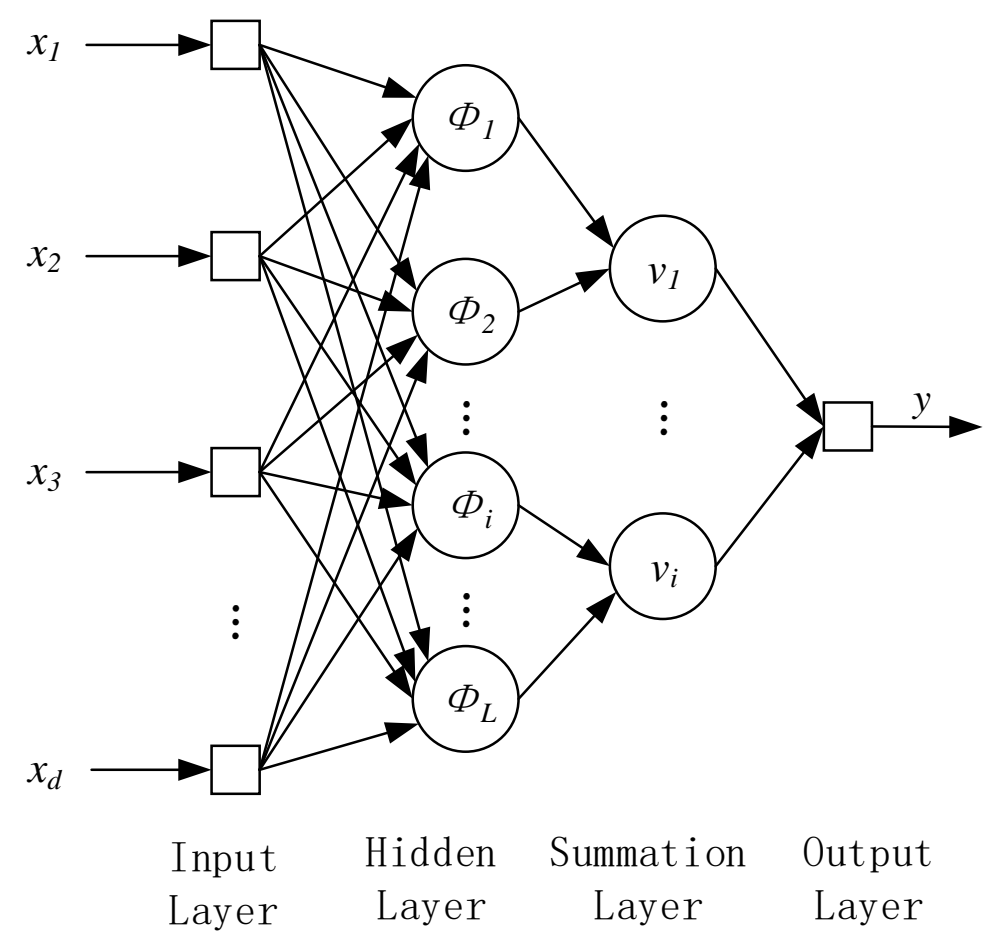

Figure 2. The Structure of PNN

The hidden layer is radial base layer, each hidden layer neuron has a center, and the layer receives the sample input from the input layer, then compute the distance between input vector and the center, finally a scalar value can be returned. The number of neurons and input training samples is the same. Vector $x$ inputs to the hidden layer, the inputloutput relationship identified by the hidden layer of the $i$ model $j$ neurons is defined as the following:

$$
\Phi_{i j}(x)=\frac{1}{(2 \pi)^{\frac{1}{2}} \sigma^{d}} e^{-\frac{\left(x-x_{i j}\right)\left(x-x_{i j}\right)^{\prime}}{\sigma^{2}}}, i=1,2, \cdots, M
$$

Where $M$ is the total number of classes in the training sample, $d$ is the dimension of the sample space data, and $x_{i j}$ is the $j$ center of the $i$ sample.

Summation layer performs a weighted average of the output of the same kind of hidden neurons in the hidden layer: 


$$
v_{i}=\frac{\sum_{j=1}^{L} \Phi_{i j}}{L}
$$

Where $v_{i}$ represents the output of $i$ category, and $L$ represents the number of neurons in $i$ category. The number of neurons in the summation layer is the same as that of the Category number $M$.

The output layer takes the maximum of the summation layer as output category:

$$
y=\arg \max \left(v_{i}\right)
$$

The method for assessing the power cable condition is divided into four states, hence, the summation layer of the PNN network contains 4 neurons. For the convenience of network computing, the output state value $1,2,3,4$ of $v_{1} 、 v_{2} 、 v_{3} 、 v_{4}$, respectively, are used to represent the normal, attention, abnormal and serious four different states.

The advantage of PNN network is that the nonlinear learning algorithm is used to complete the work of nonlinear learning algorithm, and the high precision of nonlinear algorithm is guaranteed. PNN is mainly used for classification and pattern recognition, in which the classification is most widely used.

\subsection{Comprehensive Assessment Method for Power Cable Condition}

According to the principle of factor analysis, power cable multi-source information database is analyzed, and the main factor representing the comprehensive condition of power cable are extracted. The comprehensive assessment method for power cable is proposed based on the PNN. Specific assessment steps can be summarized as follows:

(1) Whether the state variables of condition assessment are complete or not can be determined by the established power cable multi-source information database.

(2) The factor analysis can be used directly for complete information of the state variables, and the number of state variable is extracted as the main factor to characterize the condition information of power cable, and the main factor of the database is obtained under the complete information.

According to the factor analysis method on Section 2.2, the missing state variables are removed from multi-source information database under incomplete information, and the remaining state variables can get database main factor score by using factor analysis under incomplete information.

(3) Input the main factor values of the step (2) into PNN to train the neural network, which can establish the neural network for the assessment of power cable condition.

(4) Input the condition assessment of the neural network established above, we can get the results of this condition assessment. The assessment flow chart is shown in Figure 3. 


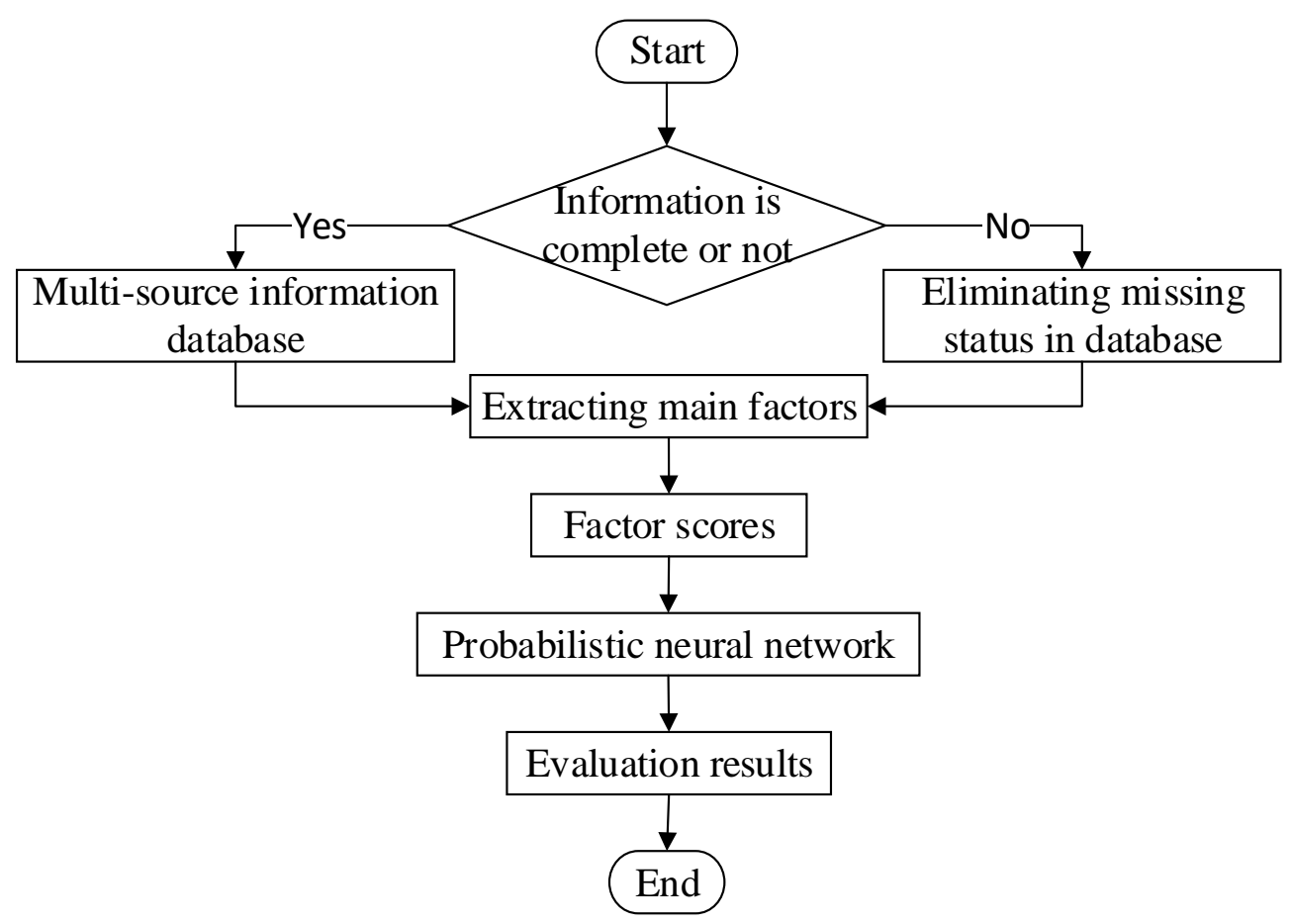

Figure 3. Flow Chart of Cable State Evaluation

\section{Case Study}

The degree of incomplete information is different for the power cable condition assessment according to the different types and number of missing state variables. To verify the scientific and effectiveness of the proposed method, first, in order to verify the effectiveness of the methods, the power cable condition under complete information in test samples is used to assess the power cable under mild incomplete information; then the degree of incomplete information is extended to severe cases to verify the scientific nature of the method.

\subsection{Condition Assessment under Mild Information Incomplete}

Mild incomplete information for power cable condition assessment refers to the amount of the missing state variables is not more than five. The detailed process is shown as follows.

\subsubsection{Determine the Multi-source Information Database}

Firstly, the missing status must be removed from the database that is established in the second section. Secondly, 100 groups power cable state history information are selected as the original sample from the database, each group contains 22 power cable condition information and the corresponding state values. The former 80 samples are taken as training samples, the latter 20 groups of samples are regarded as the test samples.

\subsubsection{Calculate the Factor Scores}

Factor scores can be calculated according to the introduction of Section 3. Firstly, as is shown in Equation (4), we can find the factor load matrix with the covariance matrix of the original data. Each state is represented through a linear function of the main factor, as shown in Table 2. 
Table 2. The Factor Score Matrix under Mild Condition of Information Incomplete

\begin{tabular}{|c|c|c|c|c|c|c|c|}
\hline \multirow{2}{*}{ State Parameter $\quad$ Main Factor } & \multicolumn{7}{|c|}{ Main Factor } \\
\hline & 1 & 2 & 3 & 4 & 5 & 6 & 7 \\
\hline Rated voltage & .186 & .029 & -.015 & -.040 & -.039 & .057 & .033 \\
\hline Rate & -.124 & .384 & .014 & -.053 & .002 & .064 & -.002 \\
\hline Length of line & .321 & -.216 & .042 & -.124 & -.047 & .011 & .031 \\
\hline Wire & .234 & -.071 & -.024 & -.010 & .030 & .089 & .004 \\
\hline Operating voltage & .186 & .027 & -.016 & -.037 & -.035 & .058 & .031 \\
\hline Opera & -.115 & .382 & .044 & -.073 & -.052 & .020 & -.044 \\
\hline Lo & -.026 & .250 & -.029 & -.062 & -.088 & -.057 & .000 \\
\hline Opera & -.094 & -.001 & .157 & .228 & .131 & -.159 & .288 \\
\hline Partial di & -.058 & .199 & -.016 & .227 & -.224 & .179 & -.023 \\
\hline Diel & -.032 & -.004 & .039 & -.007 & -.049 & -.419 & .153 \\
\hline $\begin{array}{r}\text { Conductor tempe } \\
\mathrm{ca}\end{array}$ & .053 & -.036 & .032 & .255 & .123 & -.033 & .178 \\
\hline $\begin{array}{r}\text { Insulation resist } \\
\text { insul }\end{array}$ & -.135 & .194 & -.094 & -.218 & .046 & .360 & .348 \\
\hline Insulation res & .159 & -.097 & -.213 & -.105 & .372 & .256 & .006 \\
\hline Appea & .026 & -.147 & -.200 & .470 & .134 & .060 & -.052 \\
\hline Cable j & & .077 & -.060 & .105 & -.018 & .117 & -.009 \\
\hline Sheath protection side curre & -.107 & .058 & .013 & .134 & .403 & .103 & -.115 \\
\hline The & -.050 & .032 & .037 & .056 & .073 & -.114 & -.570 \\
\hline Environn & .071 & -.004 & .041 & .014 & .002 & .508 & .192 \\
\hline Environ & .031 & .067 & -.021 & -.046 & -.404 & .153 & .002 \\
\hline Similar ca & .012 & .005 & .410 & -.112 & -.061 & -.034 & -.034 \\
\hline $\begin{array}{c}\text { Manufacturer's product quality } \\
\text { statistics }\end{array}$ & .010 & .020 & .404 & -.141 & -.041 & .007 & .002 \\
\hline
\end{tabular}

Secondly, the number of main factors needs to be determined according to the cumulative contribution rate. The cumulative contribution rate represents the amount of information that can be represented by the mains factor. In general, the main factors will be able to express enough accurate information when the cumulative contribution rate exceed 85 percent.

In this case, the contribution rate for each state variables provided by the factor analysis are shown in Table 3 . We set the direct ground current as one missing state parameter. Seven main factors are obtained by using the factor analysis method to eliminate the state parameter in the multi-source information database of the cable. From Table 2, we can see that the factor analysis method can extract 22 state variables in the database by 7 main factors, and the 7 main factors can represent $86.993 \%$ of the information of original sample. 
Table 3. The Main Factors under Mild Condition of Information Incomplete

\begin{tabular}{ccccccc}
\hline \multirow{2}{*}{ Component } & \multicolumn{3}{c}{ Initial eigenvalues } & \multicolumn{3}{c}{ Extraction sums of squared loadings } \\
\cline { 2 - 7 } & Total & $\begin{array}{c}\text { \% of } \\
\text { variance }\end{array}$ & $\begin{array}{c}\text { \% of } \\
\text { cumulative }\end{array}$ & Total & $\begin{array}{c}\text { \% of } \\
\text { variance }\end{array}$ & $\begin{array}{c}\text { \% of } \\
\text { cumulative }\end{array}$ \\
\hline 1 & 7.255 & 34.547 & 34.547 & 7.255 & 34.547 & 34.547 \\
2 & 3.171 & 15.099 & 49.646 & 3.171 & 15.099 & 49.646 \\
3 & 2.261 & 10.766 & 60.413 & 2.261 & 10.766 & 60.413 \\
4 & 1.810 & 8.621 & 69.034 & 1.810 & 8.621 & 69.034 \\
5 & 1.595 & 7.596 & 76.630 & 1.595 & 7.596 & 76.630 \\
6 & 1.124 & 5.353 & 81.983 & 1.124 & 5.353 & 81.983 \\
7 & 1.052 & 5.010 & 86.993 & 1.052 & 5.010 & 86.993 \\
8 & .689 & 3.282 & 90.275 & & & \\
9 & .624 & 2.973 & 93.248 & & & \\
10 & .391 & 1.862 & 95.110 & & & \\
$\vdots$ & $\vdots$ & $\vdots$ & $\vdots$ & & & \\
$\vdots$ & $\vdots$ & $\vdots$ & $\vdots$ & & & \\
20 & .009 & .042 & 99.985 & & & \\
21 & .003 & .015 & 100.000 & & & \\
22 & .000 & .000 & 100.000 & & & \\
\hline
\end{tabular}

When the factor loading matrix and the main factors determined, factor scores in each sample can be calculated by the formula (5).

\subsubsection{Train Probabilistic Neural Network}

We can training the probabilistic neural network with the sample of the factor scores instead of the original sample. These 80 groups of samples composed of factor scores are input to the input layer. Then the neural network for the assessment of power cable condition can be established.

\subsubsection{Evaluate the Status of the Test Samples}

After the network training is finished, the 20 groups of test samples are input into the PNN network, and the assessment results under mild information incomplete condition can be obtained from the output of the PNN network as shown in Table 4.

Table 4. The Evaluation Results under Mild Condition of Incomplete Information

\begin{tabular}{ccc}
\hline Test sample number & $\begin{array}{c}\text { The cable conditions under } \\
\text { conditions of complete } \\
\text { information }\end{array}$ & The evaluation results \\
\hline 1 & 4 & 4 \\
2 & 2 & 2 \\
3 & 1 & 1 \\
4 & 1 & 1 \\
5 & 1 & 2 \\
6 & 1 & 1 \\
7 & 2 & 2 \\
8 & 1 & 1 \\
9 & 1 & 1
\end{tabular}




\begin{tabular}{lll}
10 & 2 & 2 \\
11 & 1 & 1 \\
12 & 1 & 1 \\
13 & 2 & 2 \\
14 & 1 & 1 \\
15 & 1 & 1 \\
16 & 1 & 1 \\
17 & 2 & 2 \\
18 & 3 & 3 \\
19 & 4 & 4 \\
20 & 1 & 1 \\
\hline
\end{tabular}

Also, the cable conditions under the condition of complete information are shown in Table 4. In order to verify the effectiveness of the evaluation method, the comparison between the different evaluation results, and the power cable conditions shows that only the fifth evaluation results of the test samples appear deviation, and the normal state is evaluated as the attention state, which is in the acceptable range. On the whole, the accuracy of the evaluation results is $95 \%$. The evaluation results show that the proposed method is accurate and effective in evaluating the state of power cable under incomplete information.

\subsection{Condition Assessment under Severe Incomplete Information}

Severe incomplete information in power cable condition assessment refers to the amount of the missing state variables is more than five. In this paper, we set the amount of the missing state variables is twelve, and eliminate these state parameters in the multi-source information database of the cable. The detailed procedure is the same as in Section 5.1. According to the evaluation method proposed in this paper, the evaluation results are shown in Table 5.

Table 5. The Evaluation Results under Severe Incomplete Information

\begin{tabular}{ccc}
\hline Test sample number & $\begin{array}{c}\text { The cable conditions under } \\
\text { conditions of complete } \\
\text { information }\end{array}$ & The evaluation results \\
\hline 1 & 4 & 3 \\
2 & 2 & 2 \\
3 & 1 & 1 \\
4 & 1 & 1 \\
5 & 1 & 2 \\
6 & 1 & 1 \\
7 & 2 & 2 \\
8 & 1 & 1 \\
9 & 1 & 1 \\
10 & 2 & 1 \\
11 & 1 & 1 \\
12 & 1 & 1 \\
13 & 2 & 2 \\
14 & 1 & 1 \\
15 & 1 & 1 \\
16 & 1 & 1 \\
17 & 2 & 2 \\
18 & 3 & 3 \\
20 & 4 & 3 \\
\hline
\end{tabular}


In the evaluation results, four groups of samples appeared deviation, and the accuracy rate is $80 \%$. Every cable condition is evaluated to an adjacent state in these four results which have deviations. The results show that the accuracy of the assessment condition has declined when the loss of the state variables is severe, but the deviation is not large, and this proposed method still has important significance in power cable condition assessment. According to the state assessment method proposed in this paper, the accuracy can be obtained under different degrees of incomplete information condition assessment as shown in Figure 4.

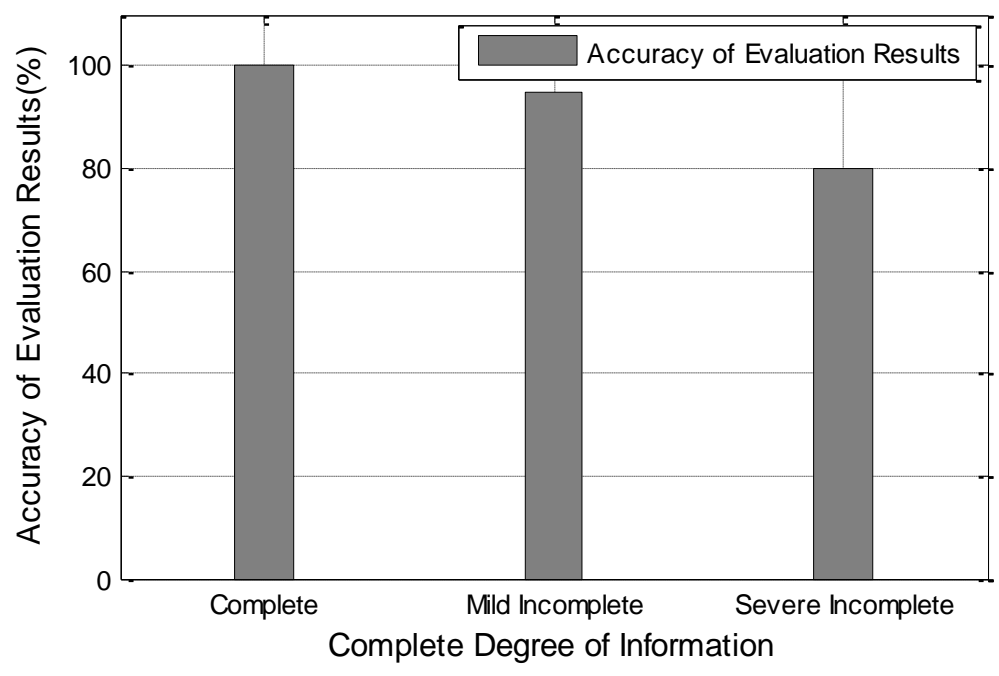

Figure 4. The Accuracy Rate of State Assessment under the Condition of Different Degree Information Incomplete

\section{Conclusions}

In this paper, the factor analysis method is used to extract the main factors of the incomplete information of power cable condition, The multi-source information database can fully reflect the operating state of the cable, is an important basis for cable status assessment. Combined with the research of this paper, the following conclusions could be obtained

1) The information about the power cable condition is divided into basic information of equipment, information operation, information retrieval and family defect, which are a total of four kinds of information. The multi-source information database can fully reflect the operating state of the cable, is an important basis for cable status assessment.

2) Factor analysis is used to extract the main factors to represent the state of the cable and to realize the fusion of the multi-source information of the cable state under the incomplete information condition, and then establish a complete cable state evaluation system, so that the application of cable state assessment method is wider.

3) Based on the probabilistic neural network, this method can overcome the influence of the subjective factors on the evaluation results and make the evaluation results more reliable. As is shown in case study, the accuracy of this method is over $80 \%$ under the incomplete information condition.

\section{References}

[1] B. Quak, E. Gulski, J. J. Smit, J. Wester and E. R. Groot, "Database support for condition assessment of distribution power cables", Electrical Insulation. Conference Record of the 2002 IEEE International 
Symposium on. IEEE, (2002), pp. 468-471.

[2] S. Thomas, "Instrumentation and concept of partial discharge and temperature cable monitoring", Power System Technology, vol. 2, (2004), pp. 1422-1427.

[3] J. J. Huan, G. Wang, H. F. Li and Z. B. Sun, "Risk Assessment of XLPE Power Cables Based on Fuzzy Comprehensive Evaluation Method", Power and Energy Engineering Conference (APPEEC), (2010), pp. $1-4$.

[4] A. B. Babaee and S. M. Shahrtash, "Cable insulation assessment by a fuzzy-based algorithm", Electrical Insulation and Dielectric Phenomena, Annual Report Conference, (2012), pp. 118-124.

[5] H. B. Sui, K. J. Li, Y. Sun and M. Liu, "Nonlinear fuzzy model for operating condition evaluation of power cables in tunnels", Industry Applications Society Annual Meeting (IAS), (2012), pp. 1-6.

[6] J. Bühler and G. Balzer, "Evaluation of the condition of medium voltage urban cable networks using fuzzy logic", Power Tech, (2009), pp. 1-8.

[7] A. A. Razi-Kazemi, M. Vakilian, K. Niayesh and M. Lehtonen, "Data Mining of Online Diagnosed Waveforms for Probabilistic Condition Assessment of SF Circuit Breakers. Power Delivery", Power Delivery, vol. 30, no. 3, (2015), pp. 1354-1362.

[8] S. Ren-jie and C. Kai, "Application of fuzzy decision tree in power cable insulation condition assessment", Advanced Computer Theory and Engineering, vol. 3, (2010), pp. V3-525.

[9] W. H. Tang, K. Spurgeon, Q. H. Wu and Z. J. Richardson, "An evidential reasoning approach to transformer condition assessments", Power Delivery, vol. 19, no. 4, (2004), pp. 1696-1703.

[10] G. Liu, G. Zhang and F. Zhou, "Aging State Assessment of $110 \mathrm{kV}$ High-voltage Cable by Isothermal Relaxation Method", High Voltage Engineering, vol. 2, (2014), pp. 025.

[11] S. Meijer, P. D. Agoris, P. P. Seitz and T. J. Hermans, "Condition assessment of power cable accessories using advanced VHF/UHF PD detection”, Electrical Insulation, (2006), pp. 482-485.

[12] E. Gulski, J. J. Smit and F. J. Wester, "PD knowledge rules for insulation condition assessment of distribution power cables", Dielectrics and Electrical Insulation, vol. 12, no. 2, (2005).

[13] W. Dong, W. Gu and K. Yang, "Research on the condition based maintenance decision of equipment in power plant. Machine Learning and Cybernetics", Machine Learning and Cybernetics, vol. 6, (2004), pp. 3468-3473.

[14] Guangdong Grid Co.Technical guidelines for equipment state evaluation and risk assessment, (2010).

[15] X. Wang, "Statistical analysis method and its application", Shanghai University of Finance and Economics press, Shang Hai, (2010).

[16] C. Ming, "MATLAB neural network principle and example", Tsinghua University press, Beijing, (2013).

\section{Authors}

Erxia Li (1984-), is a researcher in the power distribution department of China Electric Power Research Institute. Her major field of study is power distribution system condition-based maintenance and power distribution automation. E-mail: lierxia@epri.sgcc.com.cn

Yonghua FAN(1974-), is a researcher in the power distribution department of China Electric Power Research Institute. His major field of study is power distribution system condition-based maintenance and power distribution automation. E-mail: fanyonghua@epri.sgcc.com.cn

Chaoqun Kang:(1991-), is a researcher in the power distribution department of China Electric Power Research Institute. Her major field of study is power distribution system condition-based maintenance. E-mail: kangchaoqun@epri.sgcc.com.cn

Yuling Li (1985-), is a researcher in the power distribution department of China Electric Power Research Institute. His major field of study is power distribution system condition-based maintenance and power distribution automation. E-mail: liyuling@epri.sgcc.com.cn

Xinrui Hao(1991-), is a master in the school of electrical engineering of Wuhan University. His major field of study is power distribution system condition-based maintenance and power distribution automation. E-mail: haoxinrui2009@163.com 
Zheng Zhang(1993-), is a master in the school of electrical engineering of Wuhan University. His major field of study is power distribution system condition-based maintenance and power distribution automation. E-mail: whu_zhangzheng@163.com. 